\title{
A new extragradient algorithm for split equilibrium problems and fixed point problems
}

\author{
Narin Petrot ${ }^{1,2}$, Mohsen Rabbani $^{3}$, Manatchanok Khonchaliew ${ }^{1}$ and Vahid Dadashi ${ }^{3 *}$
}

\section{"Correspondence:}

vahid.dadashi@iausari.ac.ir

${ }^{3}$ Department of Mathematics, Sari Branch, Islamic Azad University, Sari, Iran

Full list of author information is available at the end of the article

\begin{abstract}
In this paper, we present a new extragradient algorithm for approximating a solution of the split equilibrium problems and split fixed point problems. The strong convergence theorems are proved in the framework of Hilbert spaces under some mild conditions. We apply the obtained main result for the problem of finding a solution of split variational inequality problems and split fixed point problems and a numerical example and computational results are also provided.
\end{abstract}

MSC: 68W10;65K10;65K15;47H09

Keywords: Split equilibrium problem; Pseudomonotonicity; Extragradient method

\section{Introduction}

Let $C$ and $D$ be nonempty closed and convex subsets of real Hilbert spaces $H_{1}$ and $H_{2}$, respectively, and let $H_{1}$ and $H_{2}$ be endowed with an inner product $\langle\cdot, \cdot\rangle$ and the corresponding norm $\|\cdot\|$. By $\rightarrow$ and $\rightarrow$, we denote strong convergence and weak convergence, respectively. Suppose that $f: C \times C \rightarrow \mathbb{R}$ be a bifunction. The equilibrium problem (EP) is to find $z \in C$ such that

$$
f(z, x) \geq 0, \quad \forall x \in C
$$

The solution set of the equilibrium problem is denoted by $\mathrm{EP}(f)$. The equilibrium problem is a generalization of many mathematical models such as variational inequalities, fixed point problems, and optimization problems; see [6, 14, 17, 18, 20, 35]. In 2013, Anh [2] introduced an extragradient algorithm for finding a common element of fixed point set of a nonexpansive mapping and solution set of an equilibrium problem on pseudomonotone and Lipschitz-type continuous bifunction in real Hilbert space. The author proved the strong convergence of the generated sequence under some condition on it. Since then, many authors considered the EP and related problems and proved weak and strong convergence. See, for example [1-4, 11, 21, 26, 41].

Moudafi [32] (see also He [25]) introduced the split equilibrium problem (SEP) which is to find $z \in C$ such that

$$
z \in \operatorname{EP}(f) \cap L^{-1}(\operatorname{EP}(g))
$$

(c) The Author(s) 2019. This article is distributed under the terms of the Creative Commons Attribution 4.0 International License (http://creativecommons.org/licenses/by/4.0/), which permits unrestricted use, distribution, and reproduction in any medium, provided you give appropriate credit to the original author(s) and the source, provide a link to the Creative Commons license, and indicate if changes were made. 
where $L: H_{1} \rightarrow H_{2}$ is a bounded linear operator and $g: D \times D \rightarrow \mathbb{R}$ be another bifunction. It is well known that SEP is a generalization of equilibrium problem by considering $g=0$ and $D=H_{2}$.

$\mathrm{He}[25]$ used the proximal method and introduced an iterative method and showed that the generated sequence converges weakly to a solution of SEP under suitable conditions on parameters provided that $f, g$ are monotone bifunctions on $C$ and $D$, respectively.

Problem SEP is an extension of many mathematical models which have been considered and studied intensively by several authors recently: split variational inequality problems [12], split common fixed point problems [7, 13, 16, 19, 28, 31, 36, 38-40], and the split feasibility problems which have been used for studying medical image reconstruction, sensor networks, intensity modulated radiation therapy, and data compression; see $[5,8-10]$ and the references quoted therein.

In this paper, motivated and inspired by the above literature, we consider a new extragradient algorithm for finding a common solution of split equilibrium problem of pseudomonotone and Lipschitz-type continuous bifunctions and split fixed point problem of nonexpansive mappings in real Hilbert space. That is, we are interested in considering the following problem: let $H_{1}$ and $H_{2}$ be real Hilbert spaces and $C$ and $D$ be nonempty closed and convex subsets of $H_{1}$ and $H_{2}$, respectively. Let $f: C \times C \rightarrow \mathbb{R}$ and $g: D \times D \rightarrow \mathbb{R}$ be pseudomonotone and Lipschitz-type continuous bifunctions, $T: C \rightarrow C$ and $S: D \rightarrow D$ be nonexpansive mappings and $L: H_{1} \rightarrow H_{2}$ be a bounded linear operator, we consider the problem of finding a solution $p \in C$ such that

$$
p \in(\mathrm{EP}(f) \cap F(T)) \cap L^{-1}(\mathrm{EP}(g) \cap F(S))=: \Omega,
$$

where $F(T)$ is the fixed points set of $T$ and $\Omega \neq \emptyset$. Under some mild conditions, the strong convergence theorem will be provided.

The paper is organized as follows. Section 2 gathers some definitions and lemmas of geometry of real Hilbert spaces and monotone bifunctions, which will be needed in the remaining sections. In Sect. 3, we prepare a new extragradient algorithm and prove the strong convergence theorem. In Sect. 4, the results of Sect. 3 are applied to solve split variational inequality problems and split fixed point problem of nonexpansive mappings. Finally, in Sect. 5, the numerical experiments are showed and discussed.

\section{Preliminaries}

We now provide some basic concepts, definitions and lemmas which will be used in the sequel. Let $C$ be a closed and convex subset of a real Hilbert space $H$. The operator $P_{C}$ is called a metric projection operator if it assigns to each $x \in H$ its nearest point $y \in C$ such that

$$
\|x-y\|=\min \{\|x-z\|: z \in C\} .
$$

An element $y$ is called the metric projection of $x$ onto $C$ and denoted by $P_{C} x$. It exists and is unique at any point of the real Hilbert space. It is well known that the metric projection operator $P_{C}$ is continuous. 
Lemma 2.1 Let $H$ is a real Hilbert space and $C$ is a nonempty, closed and convex subset of $H$. Then, for all $x \in H$, the element $z=P_{C} x$ if and only if

$$
\langle x-z, z-y\rangle \geq 0, \quad \forall y \in C .
$$

The metric projection satisfies in the following inequality:

$$
\left\|P_{C} x-P_{C} y\right\|^{2} \leq\left\langle P_{C} x-P_{C} y, x-y\right\rangle, \quad \forall x, y \in H
$$

therefore the metric projection is firmly nonexpansive operator in $H$. For more information concerning the metric projection, please see Sect. 3 of [24].

Lemma 2.2 ([23]) Let $H$ be a real Hilbert space and $T: H \rightarrow H$ be a nonexpansive mapping with $F(T) \neq \emptyset$. Then the mapping $I-T$ is demiclosed at zero, that is, if $\left\{x_{n}\right\}$ is a sequence in $H$ such that $x_{n} \rightarrow x$ and $\left\|x_{n}-T x_{n}\right\| \rightarrow 0$, then $x \in F(T)$.

Lemma 2.3 ([42]) Assume that $\left\{a_{n}\right\}$ is a sequence of nonnegative numbers such that

$$
a_{n+1} \leq\left(1-\gamma_{n}\right) a_{n}+\gamma_{n} \delta_{n}, \quad \forall n \in \mathbb{N}
$$

where $\left\{\gamma_{n}\right\}$ is a sequence in $(0,1)$ and $\left\{\delta_{n}\right\}$ is a sequence in $\mathbb{R}$ such that

(i) $\lim _{n \rightarrow \infty} \gamma_{n}=0, \sum_{n=1}^{\infty} \gamma_{n}=\infty$,

(ii) $\lim \sup _{n \rightarrow \infty} \delta_{n} \leq 0$.

Then $\lim _{n \rightarrow \infty} a_{n}=0$.

Lemma 2.4 ([30]) Let $\left\{a_{n}\right\}$ be a sequence of real numbers such that there exists a subsequence $\left\{n_{i}\right\}$ of $\{n\}$ such that $a_{n_{i}}<a_{n_{i}+1}$ for all $i \in \mathbb{N}$. Then there exists a nondecreasing sequence $\left\{m_{k}\right\} \subset \mathbb{N}$ such that $m_{k} \rightarrow \infty$ as $k \rightarrow \infty$ and the following properties are satisfied by all (sufficiently large) numbers $k \in \mathbb{N}$ :

$$
a_{m_{k}} \leq a_{m_{k}+1} \text { and } a_{k} \leq a_{m_{k}+1}
$$

In fact, $m_{k}=\max \left\{j \leq k: a_{j}<a_{j+1}\right\}$.

Definition 2.5 A bifunction $f: C \times C \rightarrow \mathbb{R}$ is said to be

- monotone on $C$ if

$$
f(x, y)+f(y, x) \leq 0, \quad \forall x, y \in C
$$

- pseudomonotone on $C$ if

$$
f(x, y) \geq 0 \quad \Longrightarrow \quad f(y, x) \leq 0, \quad \forall x, y \in C ;
$$

- Lipschitz-type continuous on $C$ if there exist two positive constants $c_{1}$ and $c_{2}$ such that

$$
f(x, y)+f(y, z) \geq f(x, z)-c_{1}\|x-y\|^{2}-c_{2}\|y-z\|^{2}, \quad \forall x, y, z \in C .
$$


Let $C$ be a nonempty closed and convex subset of a real Hilbert space $H$ and $f: C \times C \rightarrow$ $\mathbb{R}$ be a bifunction, we will assume the following conditions:

(A1) $f$ is pseudomonotone on $C$ and $f(x, x)=0$ for all $x \in C$;

(A2) $f$ is weakly continuous on $C \times C$ in the sense that if $x, y \in C$ and $\left\{x_{n}\right\},\left\{y_{n}\right\} \subset C$ converge weakly to $x$ and $y$, respectively, then $f\left(x_{n}, y_{n}\right) \rightarrow f(x, y)$ as $n \rightarrow \infty$;

(A3) $f(x, \cdot)$ is convex and subdifferentiable on $C$ for every fixed $x \in C$;

(A4) $f$ is Lipschitz-type continuous on $C$ with two positive constants $c_{1}$ and $c_{2}$.

It is easy to show that under assumptions (A1)-(A3), the solution set $\mathrm{EP}(f)$ is closed and convex (see, for instance [34]).

We need the following lemma to prove our main results.

Lemma 2.6 ([2]) Assume that $f$ satisfies (A1), (A3), (A4) such that $\mathrm{EP}(f)$ is nonempty and $0<\rho_{0}<\min \left\{\frac{1}{2 c_{1}}, \frac{1}{2 c_{2}}\right\}$. If $x_{0} \in C$, and $y_{0}, z_{0}$ are defined by

$$
\left\{\begin{array}{l}
y_{0}=\arg \min \left\{\rho_{0} f\left(x_{0}, y\right)+\frac{1}{2}\left\|y-x_{0}\right\|^{2}: y \in C\right\}, \\
z_{0}=\arg \min \left\{\rho_{0} f\left(y_{0}, y\right)+\frac{1}{2}\left\|y-x_{0}\right\|^{2}: y \in C\right\},
\end{array}\right.
$$

then

(i) $\rho_{0}\left[f\left(x_{0}, y\right)-f\left(x_{0}, y_{0}\right)\right] \geq\left\langle y_{0}-x_{0}, y_{0}-y\right\rangle, \forall y \in C$;

(ii) $\left\|z_{0}-p\right\|^{2} \leq\left\|x_{0}-p\right\|^{2}-\left(1-2 \rho_{0} c_{1}\right)\left\|x_{0}-y_{0}\right\|^{2}-\left(1-2 \rho_{0} c_{2}\right)\left\|y_{0}-z_{0}\right\|^{2}, \forall p \in \operatorname{EP}(f)$.

\section{Main results}

In this section, we present our main algorithm and show the strong convergence theorem for finding a common solution of split equilibrium problem of pseudomonotone and Lipschitz-type continuous bifunctions and split fixed point problem of nonexpansive mappings in real Hilbert space.

Let $H_{1}$ and $H_{2}$ be two real Hilbert spaces and $C$ and $D$ be nonempty closed and convex subsets of $H_{1}$ and $H_{2}$, respectively. Suppose that $f: C \times C \rightarrow \mathbb{R}$ and $g: D \times D \rightarrow \mathbb{R}$ be bifunctions. Let $L: H_{1} \rightarrow H_{2}$ be a bounded linear operator with its adjoint $L^{*}, T: C \rightarrow C$ and $S: D \rightarrow D$ be nonexpansive mappings and $h: C \rightarrow C$ be a $\rho$-contraction mapping. We introduce the following extragradient algorithm for solving the split equilibrium problem and fixed point problem.

Algorithm 3.1 Choose $x_{1} \in H_{1}$. The control parameters $\lambda_{n}, \mu_{n}, \alpha_{n}, \beta_{n}, \delta_{n}$ satisfy the following conditions:

$$
\begin{aligned}
& 0<\underline{\lambda} \leq \lambda_{n} \leq \bar{\lambda}<\min \left\{\frac{1}{2 c_{1}}, \frac{1}{2 c_{2}}\right\}, \quad 0<\underline{\mu} \leq \mu_{n} \leq \bar{\mu}<\min \left\{\frac{1}{2 d_{1}}, \frac{1}{2 d_{2}}\right\}, \\
& \beta_{n} \in(0,1), \quad 0<\liminf _{n \rightarrow \infty} \beta_{n} \leq \limsup _{n \rightarrow \infty} \beta_{n}<1, \quad 0<\underline{\delta} \leq \delta_{n} \leq \bar{\delta}<\frac{1}{\|L\|^{2}}, \\
& \alpha_{n} \in\left(0, \frac{1}{2-\rho}\right), \quad \lim _{n \rightarrow \infty} \alpha_{n}=0, \quad \sum_{n=1}^{\infty} \alpha_{n}=\infty .
\end{aligned}
$$


Let $\left\{x_{n}\right\}$ be a sequence generated by

$$
\left\{\begin{array}{l}
u_{n}=\arg \min \left\{\mu_{n} g\left(P_{D}\left(L x_{n}\right), u\right)+\frac{1}{2}\left\|u-P_{D}\left(L x_{n}\right)\right\|^{2}: u \in D\right\}, \\
v_{n}=\arg \min \left\{\mu_{n} g\left(u_{n}, u\right)+\frac{1}{2}\left\|u-P_{D}\left(L x_{n}\right)\right\|^{2}: u \in D\right\}, \\
y_{n}=P_{C}\left(x_{n}+\delta_{n} L^{*}\left(S v_{n}-L x_{n}\right)\right), \\
t_{n}=\arg \min \left\{\lambda_{n} f\left(y_{n}, y\right)+\frac{1}{2}\left\|y-y_{n}\right\|^{2}: y \in C\right\}, \\
z_{n}=\arg \min \left\{\lambda_{n} f\left(t_{n}, y\right)+\frac{1}{2}\left\|y-y_{n}\right\|^{2}: y \in C\right\}, \\
x_{n+1}=\alpha_{n} h\left(x_{n}\right)+\left(1-\alpha_{n}\right)\left(\beta_{n} x_{n}+\left(1-\beta_{n}\right) T z_{n}\right) .
\end{array}\right.
$$

Theorem 3.2 Let $H_{1}$ and $H_{2}$ be two real Hilbert spaces and $C$ and $D$ be nonempty closed and convex subsets of $H_{1}$ and $H_{2}$, respectively. Suppose that $f: C \times C \rightarrow \mathbb{R}$ and $g: D \times D \rightarrow$ $\mathbb{R}$ be bifunctions which satisfy (A1)-(A4) with some positive constants $\left\{c_{1}, c_{2}\right\}$ and $\left\{d_{1}, d_{2}\right\}$, respectively. Let $L: H_{1} \rightarrow H_{2}$ be a bounded linear operator with its adjoint $L^{*}, T: C \rightarrow C$ and $S: D \rightarrow D$ be nonexpansive mappings, $h: C \rightarrow C$ be a $\rho$-contraction mapping and $\Omega \neq \emptyset$. Then the sequence $\left\{x_{n}\right\}$ generated by Algorithm 3.1 converges strongly to $q=P_{\Omega} h(q)$.

Proof Let $p \in \Omega$. So, $p \in \mathrm{EP}(f) \cap F(T) \subset C$ and $L p \in \mathrm{EP}(g) \cap F(S) \subset D$. Since $P_{D}$ is firmly nonexpansive, we get

$$
\begin{aligned}
\left\|P_{D}\left(L x_{n}\right)-L p\right\|^{2} & =\left\|P_{D}\left(L x_{n}\right)-P_{D}(L p)\right\|^{2} \\
& \leq\left\langle P_{D}\left(L x_{n}\right)-P_{D}(L p), L x_{n}-L p\right\rangle \\
& =\left\langle P_{D}\left(L x_{n}\right)-L p, L x_{n}-L p\right\rangle \\
& =\frac{1}{2}\left[\left\|P_{D}\left(L x_{n}\right)-L p\right\|^{2}+\left\|L x_{n}-L p\right\|^{2}-\left\|P_{D}\left(L x_{n}\right)-L x_{n}\right\|^{2}\right],
\end{aligned}
$$

and hence

$$
\left\|P_{D}\left(L x_{n}\right)-L p\right\|^{2} \leq\left\|L x_{n}-L p\right\|^{2}-\left\|P_{D}\left(L x_{n}\right)-L x_{n}\right\|^{2} .
$$

Since $S$ is nonexpansive, $L p \in F(S)$ and using Lemma 2.6 and the definition of $u_{n}$ and $v_{n}$, we have

$$
\begin{aligned}
\left\|S v_{n}-L p\right\|^{2}= & \left\|S v_{n}-S(L p)\right\|^{2} \\
\leq & \left\|v_{n}-L p\right\|^{2} \\
\leq & \left\|P_{D}\left(L x_{n}\right)-L p\right\|^{2}-\left(1-2 \mu_{n} d_{1}\right)\left\|P_{D}\left(L x_{n}\right)-u_{n}\right\|^{2} \\
& -\left(1-2 \mu_{n} d_{2}\right)\left\|u_{n}-v_{n}\right\|^{2},
\end{aligned}
$$

for each $n \in \mathbb{N}$. From (3.1), (3.2) and the assumptions, we obtain

$$
\left\|S v_{n}-L p\right\|^{2} \leq\left\|L x_{n}-L p\right\|^{2}-\left\|P_{D}\left(L x_{n}\right)-L x_{n}\right\|^{2} .
$$


By (3.3), we get

$$
\begin{aligned}
\left\langle L\left(x_{n}-p\right), S v_{n}-L x_{n}\right\rangle & =\left\langle S v_{n}-L p, S v_{n}-L x_{n}\right\rangle-\left\|S v_{n}-L x_{n}\right\|^{2} \\
& =\frac{1}{2}\left[\left\|S v_{n}-L p\right\|^{2}-\left\|L x_{n}-L p\right\|^{2}-\left\|S v_{n}-L x_{n}\right\|^{2}\right] \\
& \leq-\frac{1}{2}\left\|P_{D}\left(L x_{n}\right)-L x_{n}\right\|^{2}-\frac{1}{2}\left\|S v_{n}-L x_{n}\right\|^{2} .
\end{aligned}
$$

This implies that

$$
\begin{aligned}
2 \delta_{n}\left\langle L\left(x_{n}-p\right), S v_{n}-L x_{n}\right\rangle \leq & -\delta_{n}\left\|P_{D}\left(L x_{n}\right)-L x_{n}\right\|^{2} \\
& -\delta_{n}\left\|S v_{n}-L x_{n}\right\|^{2} .
\end{aligned}
$$

Since $P_{C}$ is nonexpansive and by (3.4), we obtain

$$
\begin{aligned}
\left\|y_{n}-p\right\|^{2} & =\left\|P_{C}\left(x_{n}+\delta_{n} L^{*}\left(S v_{n}-L x_{n}\right)\right)-P_{C}(p)\right\|^{2} \\
& \leq\left\|\left(x_{n}-p\right)+\delta_{n} L^{*}\left(S v_{n}-L x_{n}\right)\right\|^{2} \\
& =\left\|x_{n}-p\right\|^{2}+\delta_{n}^{2}\left\|L^{*}\left(S v_{n}-L x_{n}\right)\right\|^{2}+2 \delta_{n}\left\langle x_{n}-p, L^{*}\left(S v_{n}-L x_{n}\right)\right\rangle \\
& \leq\left\|x_{n}-p\right\|^{2}+\delta_{n}^{2}\|L\|^{2}\left\|S v_{n}-L x_{n}\right\|^{2}-\delta_{n}\left\|P_{D}\left(L x_{n}\right)-L x_{n}\right\|^{2}-\delta_{n}\left\|S v_{n}-L x_{n}\right\|^{2} \\
& =\left\|x_{n}-p\right\|^{2}-\delta_{n}\left(1-\delta_{n}\|L\|^{2}\right)\left\|S v_{n}-L x_{n}\right\|^{2}-\delta_{n}\left\|P_{D}\left(L x_{n}\right)-L x_{n}\right\|^{2}
\end{aligned}
$$

then we obtain

$$
\left\|y_{n}-p\right\| \leq\left\|x_{n}-p\right\|
$$

By Lemma 2.6, the definition of $t_{n}$ and $z_{n}$ and the assumptions we have

$$
\left\|z_{n}-p\right\| \leq\left\|y_{n}-p\right\|
$$

for each $n \in \mathbb{N}$. From (3.6) and (3.7), we get

$$
\left\|z_{n}-p\right\| \leq\left\|x_{n}-p\right\|
$$

Set $q_{n}=\beta_{n} x_{n}+\left(1-\beta_{n}\right) T z_{n}$. It follows from (3.8) that

$$
\begin{aligned}
\left\|q_{n}-p\right\| & \leq \beta_{n}\left\|x_{n}-p\right\|+\left(1-\beta_{n}\right)\left\|T z_{n}-p\right\| \\
& \leq \beta_{n}\left\|x_{n}-p\right\|+\left(1-\beta_{n}\right)\left\|z_{n}-p\right\| \\
& \leq\left\|x_{n}-p\right\| .
\end{aligned}
$$

By the definition of $x_{n+1}$ and (3.9), we obtain

$$
\begin{aligned}
\left\|x_{n+1}-p\right\| & \leq \alpha_{n}\left\|h\left(x_{n}\right)-p\right\|+\left(1-\alpha_{n}\right)\left\|q_{n}-p\right\| \\
& \leq \alpha_{n}\left\|h\left(x_{n}\right)-h(p)\right\|+\alpha_{n}\|h(p)-p\|+\left(1-\alpha_{n}\right)\left\|x_{n}-p\right\|
\end{aligned}
$$




$$
\begin{aligned}
& \leq \alpha_{n} \rho\left\|x_{n}-p\right\|+\alpha_{n}\|h(p)-p\|+\left(1-\alpha_{n}\right)\left\|x_{n}-p\right\| \\
& \leq\left(1-\alpha_{n}(1-\rho)\right)\left\|x_{n}-p\right\|+\alpha_{n}(1-\rho) \frac{\|h(p)-p\|}{1-\rho} \\
& \leq \max \left\{\left\|x_{n}-p\right\|, \frac{\|h(p)-p\|}{1-\rho}\right\} \\
& \vdots \\
& \leq \max \left\{\left\|x_{1}-p\right\|, \frac{\|h(p)-p\|}{1-\rho}\right\} .
\end{aligned}
$$

This implies that the sequence $\left\{x_{n}\right\}$ is bounded. By (3.6) and (3.8), the sequences $\left\{y_{n}\right\}$ and $\left\{z_{n}\right\}$ are bounded too.

By Lemma 2.6, (3.6), the definition of $q_{n}$ and assumptions on $\beta_{n}$ and $\delta_{n}$, we get

$$
\begin{aligned}
\left\|q_{n}-p\right\|^{2} \leq & \beta_{n}\left\|x_{n}-p\right\|^{2}+\left(1-\beta_{n}\right)\left\|T z_{n}-p\right\|^{2} \\
\leq & \beta_{n}\left\|x_{n}-p\right\|^{2}+\left(1-\beta_{n}\right)\left\|z_{n}-p\right\|^{2} \\
\leq & \beta_{n}\left\|x_{n}-p\right\|^{2}+\left(1-\beta_{n}\right) \\
& \times\left[\left\|y_{n}-p\right\|^{2}-\left(1-2 \lambda_{n} c_{1}\right)\left\|y_{n}-t_{n}\right\|^{2}-\left(1-2 \lambda_{n} c_{2}\right)\left\|t_{n}-z_{n}\right\|^{2}\right] \\
\leq & \beta_{n}\left\|x_{n}-p\right\|^{2}+\left(1-\beta_{n}\right) \\
& \times\left[\left\|x_{n}-p\right\|^{2}-\left(1-2 \lambda_{n} c_{1}\right)\left\|y_{n}-t_{n}\right\|^{2}-\left(1-2 \lambda_{n} c_{2}\right)\left\|t_{n}-z_{n}\right\|^{2}\right] \\
= & \left\|x_{n}-p\right\|^{2}-\left(1-\beta_{n}\right)\left[\left(1-2 \lambda_{n} c_{1}\right)\left\|y_{n}-t_{n}\right\|^{2}+\left(1-2 \lambda_{n} c_{2}\right)\left\|t_{n}-z_{n}\right\|^{2}\right] .
\end{aligned}
$$

Therefore,

$$
\begin{aligned}
\left\|x_{n+1}-p\right\|^{2} \leq & \alpha_{n}\left\|h\left(x_{n}\right)-p\right\|^{2}+\left(1-\alpha_{n}\right)\left\|q_{n}-p\right\|^{2} \\
\leq & \alpha_{n}\left\|h\left(x_{n}\right)-p\right\|^{2}+\left(1-\alpha_{n}\right)\left\{\left\|x_{n}-p\right\|^{2}-\left(1-\beta_{n}\right)\left[\left(1-2 \lambda_{n} c_{1}\right)\left\|y_{n}-t_{n}\right\|^{2}\right.\right. \\
& \left.\left.+\left(1-2 \lambda_{n} c_{2}\right)\left\|t_{n}-z_{n}\right\|^{2}\right]\right\}
\end{aligned}
$$

and hence

$$
\begin{aligned}
& \left(1-\beta_{n}\right)\left[\left(1-2 \lambda_{n} c_{1}\right)\left\|y_{n}-t_{n}\right\|^{2}+\left(1-2 \lambda_{n} c_{2}\right)\left\|t_{n}-z_{n}\right\|^{2}\right] \\
& \quad \leq\left\|x_{n}-p\right\|^{2}-\left\|x_{n+1}-p\right\|^{2}+\alpha_{n} M,
\end{aligned}
$$

where

$$
\begin{aligned}
M= & \sup \left\{\left|\left\|h\left(x_{n}\right)-p\right\|^{2}-\left\|x_{n}-p\right\|^{2}\right|+\left(1-\beta_{n}\right)\left[\left(1-2 \lambda_{n} c_{1}\right)\left\|y_{n}-t_{n}\right\|^{2}\right.\right. \\
& \left.\left.+\left(1-2 \lambda_{n} c_{2}\right)\left\|t_{n}-z_{n}\right\|^{2}\right], n \in \mathbb{N}\right\} .
\end{aligned}
$$

By (3.9), we have

$$
\begin{aligned}
\left\|x_{n+1}-p\right\|^{2} & =\left\|\alpha_{n}\left(h\left(x_{n}\right)-p\right)+\left(1-\alpha_{n}\right)\left(q_{n}-p\right)\right\|^{2} \\
& \leq\left(1-\alpha_{n}\right)^{2}\left\|q_{n}-p\right\|^{2}+2 \alpha_{n}\left\langle h\left(x_{n}\right)-p, x_{n+1}-p\right\rangle
\end{aligned}
$$




$$
\begin{aligned}
\leq & \left(1-\alpha_{n}\right)^{2}\left\|x_{n}-p\right\|^{2}+2 \alpha_{n}\left\langle h\left(x_{n}\right)-h(p), x_{n+1}-p\right\rangle+2 \alpha_{n}\left\langle h(p)-p, x_{n+1}-p\right\rangle \\
\leq & \left(1-\alpha_{n}\right)^{2}\left\|x_{n}-p\right\|^{2}+2 \alpha_{n} \rho\left\|x_{n}-p\right\|\left\|x_{n+1}-p\right\|+2 \alpha_{n}\left\langle h(p)-p, x_{n+1}-p\right\rangle \\
\leq & \left(1-\alpha_{n}\right)^{2}\left\|x_{n}-p\right\|^{2}+\alpha_{n} \rho\left(\left\|x_{n}-p\right\|^{2}+\left\|x_{n+1}-p\right\|^{2}\right) \\
& +2 \alpha_{n}\left\langle h(p)-p, x_{n+1}-p\right\rangle \\
= & \left(\left(1-\alpha_{n}\right)^{2}+\alpha_{n} \rho\right)\left\|x_{n}-p\right\|^{2}+\alpha_{n} \rho\left\|x_{n+1}-p\right\|^{2} \\
& +2 \alpha_{n}\left\langle h(p)-p, x_{n+1}-p\right\rangle .
\end{aligned}
$$

So, we get

$$
\begin{aligned}
\left\|x_{n+1}-p\right\|^{2} \leq & \left(1-\frac{2(1-\rho) \alpha_{n}}{1-\alpha_{n} \rho}\right)\left\|x_{n}-p\right\|^{2} \\
& +\frac{2(1-\rho) \alpha_{n}}{1-\alpha_{n} \rho}\left(\frac{\alpha_{n} M_{0}}{2(1-\rho)}+\frac{1}{(1-\rho)}\left\langle h(p)-p, x_{n+1}-p\right\rangle\right) \\
= & \left(1-\gamma_{n}\right)\left\|x_{n}-p\right\|^{2} \\
& +\gamma_{n}\left(\frac{\alpha_{n} M_{0}}{2(1-\rho)}+\frac{1}{(1-\rho)}\left\langle h(p)-p, x_{n+1}-p\right\rangle\right),
\end{aligned}
$$

where $M_{0}=\sup \left\{\left\|x_{n}-p\right\|^{2}, n \in \mathbb{N}\right\}$, put $\gamma_{n}=\frac{2(1-\rho) \alpha_{n}}{1-\alpha_{n} \rho}$ for each $n \in \mathbb{N}$. By the assumptions on $\alpha_{n}$, we have

$$
\lim _{n \rightarrow \infty} \gamma_{n}=0, \quad \sum_{n=1}^{\infty} \gamma_{n}=\infty
$$

Since $P_{\Omega} h$ is a contraction on $C$, there exists $q \in \Omega$ such that $q=P_{\Omega} h(q)$. We prove that the sequence $\left\{x_{n}\right\}$ converges strongly to $q=P_{\Omega} h(q)$. In order to prove it, let us consider two cases.

Case 1. Suppose that there exists $n_{0} \in \mathbb{N}$ such that $\left\{\left\|x_{n}-q\right\|\right\}_{n=n_{0}}^{\infty}$ is nonincreasing. In this case, the limit of $\left\{\left\|x_{n}-q\right\|\right\}$ exists. This together with the assumptions on $\left\{\alpha_{n}\right\},\left\{\beta_{n}\right\}$, $\left\{\lambda_{n}\right\}$ and (3.10) implies that

$$
\lim _{n \rightarrow \infty}\left\|y_{n}-t_{n}\right\|=\lim _{n \rightarrow \infty}\left\|t_{n}-z_{n}\right\|=0
$$

On the other hands, from the definition of $x_{n+1}$ and (3.8), we get

$$
\begin{aligned}
\left\|x_{n+1}-q\right\|^{2} \leq & \alpha_{n}\left\|h\left(x_{n}\right)-q\right\|^{2}+\left(1-\alpha_{n}\right)\left\|\beta_{n} x_{n}+\left(1-\beta_{n}\right) T z_{n}-q\right\|^{2} \\
= & \alpha_{n}\left\|h\left(x_{n}\right)-q\right\|^{2}+\left(1-\alpha_{n}\right) \\
& \times\left[\beta_{n}\left\|x_{n}-q\right\|^{2}+\left(1-\beta_{n}\right)\left\|T z_{n}-q\right\|^{2}-\beta_{n}\left(1-\beta_{n}\right)\left\|x_{n}-T z_{n}\right\|^{2}\right] \\
\leq & \alpha_{n}\left\|h\left(x_{n}\right)-q\right\|^{2}+\left(1-\alpha_{n}\right) \\
& \times\left[\beta_{n}\left\|x_{n}-q\right\|^{2}+\left(1-\beta_{n}\right)\left\|x_{n}-q\right\|^{2}-\beta_{n}\left(1-\beta_{n}\right)\left\|x_{n}-T z_{n}\right\|^{2}\right] \\
= & \alpha_{n}\left\|h\left(x_{n}\right)-q\right\|^{2}+\left(1-\alpha_{n}\right)\left[\left\|x_{n}-q\right\|^{2}-\beta_{n}\left(1-\beta_{n}\right)\left\|x_{n}-T z_{n}\right\|^{2}\right],
\end{aligned}
$$


and hence

$$
\begin{gathered}
\beta_{n}\left(1-\beta_{n}\right)\left(1-\alpha_{n}\right)\left\|x_{n}-T z_{n}\right\|^{2} \leq \alpha_{n}\left\|h\left(x_{n}\right)-q\right\|^{2}+\left\|x_{n}-q\right\|^{2} \\
-\left\|x_{n+1}-q\right\|^{2} .
\end{gathered}
$$

Since the limit of $\left\{\left\|x_{n}-q\right\|\right\}$ exists and by the assumptions on $\left\{\alpha_{n}\right\}$ and $\left\{\beta_{n}\right\}$, we obtain

$$
\lim _{n \rightarrow \infty}\left\|x_{n}-T z_{n}\right\|=0
$$

From (3.9) and (3.11), we have

$$
\begin{aligned}
\left\|x_{n+1}-q\right\|^{2}-\left\|x_{n}-q\right\|^{2}-2 \alpha_{n}\left\langle h\left(x_{n}\right)-q, x_{n+1}-q\right\rangle & \leq\left\|q_{n}-q\right\|^{2}-\left\|x_{n}-q\right\|^{2} \\
& \leq 0 .
\end{aligned}
$$

Again, since the limit of $\left\{\left\|x_{n}-q\right\|\right\}$ exists and $\alpha_{n} \rightarrow 0$, it follows that

$$
\lim _{n \rightarrow \infty}\left(\left\|q_{n}-q\right\|^{2}-\left\|x_{n}-q\right\|^{2}\right)=0
$$

and hence

$$
\lim _{n \rightarrow \infty}\left\|q_{n}-q\right\|=\lim _{n \rightarrow \infty}\left\|x_{n}-q\right\|,
$$

and by (3.9), we get

$$
\lim _{n \rightarrow \infty}\left\|x_{n}-q\right\|=\lim _{n \rightarrow \infty}\left\|z_{n}-q\right\| .
$$

We also get from (3.6), (3.7) and (3.18)

$$
\lim _{n \rightarrow \infty}\left\|x_{n}-q\right\|=\lim _{n \rightarrow \infty}\left\|y_{n}-q\right\| .
$$

By (3.5) and (3.19),

$$
\lim _{n \rightarrow \infty}\left\|S v_{n}-L x_{n}\right\|=\lim _{n \rightarrow \infty}\left\|P_{D}\left(L x_{n}\right)-L x_{n}\right\|=0,
$$

which implies that

$$
\lim _{n \rightarrow \infty}\left\|S v_{n}-P_{D}\left(L x_{n}\right)\right\|=0
$$

It follows from (3.2) that

$$
\begin{aligned}
(1 & \left.-2 \mu_{n} d_{1}\right)\left\|P_{D}\left(L x_{n}\right)-u_{n}\right\|^{2}+\left(1-2 \mu_{n} d_{2}\right)\left\|u_{n}-v_{n}\right\|^{2} \\
& \leq\left\|P_{D}\left(L x_{n}\right)-L p\right\|^{2}-\left\|S v_{n}-L p\right\|^{2} \\
& =\left(\left\|P_{D}\left(L x_{n}\right)-L p\right\|+\left\|S v_{n}-L p\right\|\right)\left(\left\|P_{D}\left(L x_{n}\right)-L p\right\|-\left\|S v_{n}-L p\right\|\right) \\
& =\left(\left\|P_{D}\left(L x_{n}\right)-L p\right\|+\left\|S v_{n}-L p\right\|\right)\left\|P_{D}\left(L x_{n}\right)-S v_{n}\right\| .
\end{aligned}
$$


So,

$$
\lim _{n \rightarrow \infty}\left\|P_{D}\left(L x_{n}\right)-u_{n}\right\|=\lim _{n \rightarrow \infty}\left\|u_{n}-v_{n}\right\|=0
$$

and hence

$$
\lim _{n \rightarrow \infty}\left\|P_{D}\left(L x_{n}\right)-v_{n}\right\|=0
$$

From (3.20) and (3.23), we get

$$
\lim _{n \rightarrow \infty}\left\|L x_{n}-v_{n}\right\|=0
$$

It follows from $x_{n} \in C$, the definition of $y_{n}$ and (3.20) that

$$
\begin{aligned}
\left\|y_{n}-x_{n}\right\| & =\left\|P_{C}\left(x_{n}+\delta_{n} L^{*}\left(S v_{n}-L x_{n}\right)\right)-P_{C}\left(x_{n}\right)\right\| \\
& \leq\left\|x_{n}+\delta_{n} L^{*}\left(S v_{n}-L x_{n}\right)-x_{n}\right\| \\
& \leq \delta_{n}\|L\|\left\|S v_{n}-L x_{n}\right\| \rightarrow 0 .
\end{aligned}
$$

Because $\left\{x_{n}\right\}$ is bounded, there exists a subsequence $\left\{x_{n_{k}}\right\}$ of $\left\{x_{n}\right\}$ such that $\left\{x_{n_{k}}\right\}$ converges weakly to some $\bar{x}$, as $k \rightarrow \infty$ and

$$
\begin{aligned}
\limsup _{n \rightarrow \infty}\left\langle x_{n}-q, h(q)-q\right\rangle & =\lim _{k \rightarrow \infty}\left\langle x_{n_{k}}-q, h(q)-q\right\rangle \\
& =\langle\bar{x}-q, h(q)-q\rangle .
\end{aligned}
$$

Consequently $\left\{L x_{n_{k}}\right\}$ converges weakly to $L \bar{x}$. By (3.24), $\left\{v_{n_{k}}\right\}$ converges weakly to $L \bar{x}$. We show that $\bar{x} \in \Omega$. We know that $x_{n} \in C$ and $v_{n} \in D$, for each $n \in \mathbb{N}$. Since $C$ and $D$ are closed and convex sets, so $C$ and $D$ are weakly closed, therefore, $\bar{x} \in C$ and $L \bar{x} \in D$. From (3.25) and (3.14), we see that $\left\{y_{n_{k}}\right\},\left\{t_{n_{k}}\right\}$ and $\left\{z_{n_{k}}\right\}$ converge weakly to $\bar{x}$. By (3.22) and (3.23), we also see that $\left\{u_{n_{k}}\right\}$ and $\left\{P_{D}\left(L x_{n_{k}}\right)\right\}$ converge weakly to $L \bar{x}$. Algorithm 3.1 and assertion (i) in Lemma 2.6 imply that

$$
\begin{aligned}
\lambda_{n_{k}}\left(f\left(y_{n_{k}}, y\right)-f\left(y_{n_{k}}, t_{n_{k}}\right)\right) & \geq\left\langle t_{n_{k}}-y_{n_{k}}, t_{n_{k}}-y\right\rangle \\
& \geq-\left\|t_{n_{k}}-y_{n_{k}}\right\|\left\|t_{n_{k}}-y\right\|, \quad \forall y \in C,
\end{aligned}
$$

and

$$
\begin{aligned}
\mu_{n_{k}}\left(g\left(P_{D}\left(L x_{n_{k}}\right), u\right)-g\left(P_{D}\left(L x_{n_{k}}\right), u_{n_{k}}\right)\right) & \geq\left\langle u_{n_{k}}-P_{D}\left(L x_{n_{k}}\right), u_{n_{k}}-u\right\rangle \\
& \geq-\left\|u_{n_{k}}-P_{D}\left(L x_{n_{k}}\right)\right\|\left\|u_{n_{k}}-u\right\|, \quad \forall u \in D .
\end{aligned}
$$

Hence, it follows that

$$
f\left(y_{n_{k}}, y\right)-f\left(y_{n_{k}}, t_{n_{k}}\right)+\frac{1}{\lambda_{n_{k}}}\left\|t_{n_{k}}-y_{n_{k}}\right\|\left\|t_{n_{k}}-y\right\| \geq 0, \quad \forall y \in C
$$


and

$$
g\left(P_{D}\left(L x_{n_{k}}\right), u\right)-g\left(P_{D}\left(L x_{n_{k}}\right), u_{n_{k}}\right)+\frac{1}{\mu_{n_{k}}}\left\|u_{n_{k}}-P_{D}\left(L x_{n_{k}}\right)\right\|\left\|u_{n_{k}}-u\right\| \geq 0, \quad \forall u \in D .
$$

Letting $k \rightarrow \infty$, by the hypothesis on $\left\{\lambda_{n}\right\},\left\{\mu_{n}\right\},(3.14),(3.22)$ and the weak continuity of $f$ and $g$ (condition (A2)), we obtain

$$
f(\bar{x}, y) \geq 0, \quad \forall y \in C \quad \text { and } \quad g(L \bar{x}, u) \geq 0, \quad \forall u \in D .
$$

This means that $\bar{x} \in \operatorname{EP}(f)$ and $L \bar{x} \in \operatorname{EP}(g)$. It follows from (3.14), (3.16) and (3.25) that

$$
\left\|z_{n}-T z_{n}\right\| \leq\left\|z_{n}-t_{n}\right\|+\left\|t_{n}-y_{n}\right\|+\left\|y_{n}-x_{n}\right\|+\left\|x_{n}-T z_{n}\right\| \rightarrow 0 .
$$

This together with Lemma 2.2 implies that $\bar{x} \in F(T)$. On the other hand, from (3.21) and (3.23), we get

$$
\left\|v_{n}-S v_{n}\right\| \leq\left\|v_{n}-P_{D}\left(L x_{n}\right)\right\|+\left\|P_{D}\left(L x_{n}\right)-S v_{n}\right\| \rightarrow 0,
$$

and using again Lemma 2.2, we obtain $L \bar{x} \in F(S)$. Then we proved that $\bar{x} \in \mathrm{EP}(f) \cap F(T)$ and $L \bar{x} \in \operatorname{EP}(g) \cap F(S)$, that is, $\bar{x} \in \Omega$. By Lemma 2.1, $\bar{x} \in \Omega$ and (3.26), we get

$$
\limsup _{n \rightarrow \infty}\left\langle x_{n}-q, h(q)-q\right\rangle=\langle\bar{x}-q, h(q)-q\rangle \leq 0 .
$$

Finally, from (3.12), (3.13), (3.27) and Lemma 2.3, we find that the sequence $\left\{x_{n}\right\}$ converges strongly to $q$.

Case 2. Suppose that there exists a subsequence $\left\{n_{i}\right\}$ of $\{n\}$ such that

$$
\left\|x_{n_{i}}-q\right\|<\left\|x_{n_{i}+1}-q\right\|, \quad \forall i \in \mathbb{N} .
$$

According to Lemma 2.4, there exists a nondecreasing sequence $\left\{m_{k}\right\} \subset \mathbb{N}$ such that $m_{k} \rightarrow$ $\infty$,

$$
\left\|x_{m_{k}}-q\right\| \leq\left\|x_{m_{k}+1}-q\right\| \quad \text { and } \quad\left\|x_{k}-q\right\| \leq\left\|x_{m_{k}+1}-q\right\|, \quad \forall k \in \mathbb{N} .
$$

From this and (3.10), we get

$$
\begin{aligned}
& \left(1-\beta_{m_{k}}\right)\left[\left(1-2 \lambda_{m_{k}} c_{1}\right)\left\|y_{m_{k}}-t_{m_{k}}\right\|^{2}+\left(1-2 \lambda_{m_{k}} c_{2}\right)\left\|t_{m_{k}}-z_{m_{k}}\right\|^{2}\right] \\
& \quad \leq \alpha_{m_{k}} M+\left\|x_{m_{k}}-q\right\|^{2}-\left\|x_{m_{k}+1}-q\right\|^{2} \\
& \quad \leq \alpha_{m_{k}} M .
\end{aligned}
$$

This together with the assumptions on $\left\{\alpha_{n}\right\},\left\{\beta_{n}\right\}$ and $\left\{\lambda_{n}\right\}$ implies that

$$
\lim _{k \rightarrow \infty}\left\|y_{m_{k}}-t_{m_{k}}\right\|=0, \quad \lim _{k \rightarrow \infty}\left\|t_{m_{k}}-z_{m_{k}}\right\|=0 \quad \text { and } \quad \lim _{k \rightarrow \infty}\left\|y_{m_{k}}-z_{m_{k}}\right\|=0 .
$$


From (3.15), we have

$$
\begin{aligned}
\beta_{m_{k}}\left(1-\beta_{m_{k}}\right)\left(1-\alpha_{m_{k}}\right)\left\|x_{m_{k}}-T z_{m_{k}}\right\|^{2} & \leq \alpha_{m_{k}}\left\|h\left(x_{m_{k}}\right)-q\right\|^{2}+\left\|x_{m_{k}}-q\right\|^{2}-\left\|x_{m_{k}+1}-q\right\|^{2} \\
& \leq \alpha_{m_{k}}\left\|h\left(x_{m_{k}}\right)-q\right\|^{2} .
\end{aligned}
$$

By the hypothesis on $\left\{\alpha_{n}\right\}$ and $\left\{\beta_{n}\right\}$, we have

$$
\lim _{k \rightarrow \infty}\left\|x_{m_{k}}-T z_{m_{k}}\right\|=0
$$

By (3.17), we get

$$
\begin{aligned}
-2 \alpha_{m_{k}}\left\langle h\left(x_{m_{k}}\right)-q, x_{m_{k}+1}-q\right\rangle \leq & \left\|x_{m_{k}+1}-q\right\|^{2}-\left\|x_{m_{k}}-q\right\|^{2} \\
& -2 \alpha_{m_{k}}\left\langle h\left(x_{m_{k}}\right)-q, x_{m_{k}+1}-q\right\rangle \\
\leq & \left\|q_{m_{k}}-q\right\|^{2}-\left\|x_{m_{k}}-q\right\|^{2} \leq 0 .
\end{aligned}
$$

Since the sequence $\left\{x_{n}\right\}$ is bounded and $\alpha_{n} \rightarrow 0$, we obtain

$$
\lim _{k \rightarrow \infty}\left\|q_{m_{k}}-q\right\|=\lim _{k \rightarrow \infty}\left\|x_{m_{k}}-q\right\|
$$

By the same argument as Case 1, we have

$$
\limsup _{k \rightarrow \infty}\left\langle x_{m_{k}}-q, h(q)-q\right\rangle \leq 0
$$

It follows from (3.12) and (3.28) that

$$
\begin{aligned}
\left\|x_{m_{k}+1}-q\right\|^{2} & \leq\left(1-\gamma_{m_{k}}\right)\left\|x_{m_{k}}-q\right\|^{2}+\gamma_{m_{k}}\left(\frac{\alpha_{m_{k}} M_{0}}{2(1-\rho)}+\frac{1}{(1-\rho)}\left\langle h(q)-q, x_{m_{k}+1}-q\right\rangle\right) \\
& \leq\left(1-\gamma_{m_{k}}\right)\left\|x_{m_{k}+1}-q\right\|^{2}+\gamma_{m_{k}}\left(\frac{\alpha_{m_{k}} M_{0}}{2(1-\rho)}+\frac{1}{(1-\rho)}\left\langle h(q)-q, x_{m_{k}+1}-q\right\rangle\right),
\end{aligned}
$$

and hence

$$
\gamma_{m_{k}}\left\|x_{m_{k}+1}-q\right\|^{2} \leq \gamma_{m_{k}}\left(\frac{\alpha_{m_{k}} M_{0}}{2(1-\rho)}+\frac{1}{(1-\rho)}\left\langle h(q)-q, x_{m_{k}+1}-q\right\rangle\right) .
$$

Since $\gamma_{m_{k}}>0$ and using (3.28) we get

$$
\left\|x_{k}-q\right\|^{2} \leq\left\|x_{m_{k}+1}-q\right\|^{2} \leq\left(\frac{\alpha_{m_{k}} M_{0}}{2(1-\rho)}+\frac{1}{(1-\rho)}\left\langle h(q)-q, x_{m_{k}+1}-q\right\rangle\right) .
$$

Taking the limit in the above inequality as $k \rightarrow \infty$, we conclude that $x_{k}$ converges strongly to $q=P_{\Omega} h(q)$.

\section{Application to variational inequality problems}

In this section, we apply Theorem 3.2 for finding a solution of a variational inequality problems for a monotone and Lipschitz-type continuous mapping. Let $H$ be a real Hilbert 
space, $C$ be a nonempty and convex subset of $H$ and $A: C \rightarrow C$ be a nonlinear operator. The mapping $A$ is said to be

- monotone on $C$ if

$$
\langle A x-A y, x-y\rangle \geq 0, \quad \forall x, y \in C
$$

- pseudomonotone on $C$ if

$$
\langle A x, y-x\rangle \geq 0 \quad \Longrightarrow \quad\langle A y, x-y\rangle \leq 0, \quad \forall x, y \in C ;
$$

- L-Lipschitz continuous on $C$ if there exists a positive constant $\mathrm{L}$ such that

$$
\|A x-A y\| \leq L\|x-y\|, \quad \forall x, y \in C .
$$

The variational inequality problem is to find $x^{*} \in C$ such that

$$
\left\langle A x^{*}, x-x^{*}\right\rangle \geq 0, \quad \forall x \in C .
$$

For each $x, y \in C$, we define $f(x, y)=\langle A x, y-x\rangle$, then the equilibrium problem (1.1) become the variational inequality problem (4.1). We denote the set of solutions of the problem (4.1) by $\operatorname{VI}(C, A)$. We assume that $A$ satisfies the following conditions:

(B1) $A$ is pseudomonotone on $C$;

(B2) $A$ is weak to strong continuous on $C$ that is, $A x_{n} \rightarrow A x$ for each sequence $\left\{x_{n}\right\} \subset C$ converging weakly to $x$;

(B3) $A$ is $\mathrm{L}_{1}$-Lipschitz continuous on $C$ for some positive constant $\mathrm{L}_{1}>0$.

Let $H_{1}$ and $H_{2}$ be two real Hilbert spaces and $C$ and $D$ be nonempty closed and convex subsets of $H_{1}$ and $H_{2}$, respectively. Suppose that $A: C \rightarrow C$ and $B: D \rightarrow D$ are $\mathrm{L}_{1}$ and $\mathrm{L}_{2}$-Lipschitz continuous on $C$ and $D$, respectively. Let $L: H_{1} \rightarrow H_{2}$ be a bounded linear operator with its adjoint $L^{*}, T: C \rightarrow C$ and $S: D \rightarrow D$ be nonexpansive mappings and $h: C \rightarrow C$ be a $\rho$-contraction mapping. We consider the following extragradient algorithm for solving the split variational inequality problems and fixed point problems.

Algorithm 4.1 Choose $x_{1} \in H_{1}$. The control parameters $\lambda_{n}, \mu_{n}, \alpha_{n}, \beta_{n}, \delta_{n}$ satisfy the following conditions:

$$
\begin{aligned}
& 0<\underline{\lambda} \leq \lambda_{n} \leq \bar{\lambda}<L_{1}, \quad 0<\underline{\mu} \leq \mu_{n} \leq \bar{\mu}<L_{2}, \quad \beta_{n} \in(0,1), \\
& 0<\liminf _{n \rightarrow \infty} \beta_{n} \leq \limsup _{n \rightarrow \infty} \beta_{n}<1, \quad 0<\underline{\delta} \leq \delta_{n} \leq \bar{\delta}<\frac{1}{\|L\|^{2}}, \\
& \alpha_{n} \in\left(0, \frac{1}{2-\rho}\right), \quad \lim _{n \rightarrow \infty} \alpha_{n}=0, \quad \sum_{n=1}^{\infty} \alpha_{n}=\infty .
\end{aligned}
$$


Let $\left\{x_{n}\right\}$ be a sequence generated by

$$
\left\{\begin{array}{l}
u_{n}=P_{D}\left(P_{D}\left(L x_{n}\right)-\mu_{n} B\left(P_{D}\left(L x_{n}\right)\right)\right), \\
\left.v_{n}=P_{D}\left(P_{D}\left(L x_{n}\right)-\mu_{n} B\left(u_{n}\right)\right)\right), \\
y_{n}=P_{C}\left(x_{n}+\delta_{n} L^{*}\left(S v_{n}-L x_{n}\right)\right), \\
t_{n}=P_{C}\left(y_{n}-\lambda_{n} A y_{n}\right), \\
z_{n}=P_{C}\left(y_{n}-\lambda_{n} A t_{n}\right), \\
x_{n+1}=\alpha_{n} h\left(x_{n}\right)+\left(1-\alpha_{n}\right)\left(\beta_{n} x_{n}+\left(1-\beta_{n}\right) T z_{n}\right) .
\end{array}\right.
$$

Theorem 4.2 Let $A: C \rightarrow C$ and $B: D \rightarrow D$ be mappings such that assumptions (B1)(B3) hold with some positive constants $\mathrm{L}_{1}>0$ and $\mathrm{L}_{2}>0$, respectively and $\Omega:=\{p \in$ $\mathrm{VI}(C, A) \cap F(T), L p \in \mathrm{VI}(D, B) \cap F(S)\} \neq \emptyset$. Then the sequence $\left\{x_{n}\right\}$ generated by Algorithm 4.1 converges strongly to $q=P_{\Omega} h(q)$.

Proof Since the mapping $A$ is satisfied the assumptions (B1)-(B3), it is easy to check that the bifunction $f(x, y)=\langle A x, y-x\rangle$ satisfies conditions (A1)-(A3). Moreover, since $A$ is $\mathrm{L}_{1}$ Lipschitz continuous on $C$, it follows that

$$
\begin{aligned}
f(x, y)+f(y, z)-f(x, z) & =\langle A x-A y, y-z\rangle \\
& \geq-\|A x-A y\|\|y-z\| \\
& \geq-L_{1}\|x-y\|\|y-z\| \\
& \geq-\frac{L_{1}}{2}\|x-y\|^{2}-\frac{L_{1}}{2}\|y-z\|^{2}, \quad \forall x, y, z \in C .
\end{aligned}
$$

Then $f$ is Lipschitz-type continuous on $C$ with $c_{1}=c_{2}=\frac{L_{1}}{2}$, and hence $f$ satisfies condition (A4).

It follows from the definitions of $f$ and $y_{n}$ that

$$
\begin{aligned}
t_{n} & =\arg \min \left\{\lambda_{n}\left\langle A y_{n}, y-y_{n}\right\rangle+\frac{1}{2}\left\|y-y_{n}\right\|^{2}: y \in C\right\} \\
& =\arg \min \left\{\frac{1}{2}\left\|y-\left(y_{n}-\lambda_{n} A y_{n}\right)\right\|^{2}: y \in C\right\} \\
& =P_{C}\left(y_{n}-\lambda_{n} A y_{n}\right),
\end{aligned}
$$

and similarly, we can get $u_{n}=P_{D}\left(P_{D}\left(L x_{n}\right)-\mu_{n} B\left(P_{D}\left(L x_{n}\right)\right)\right), v_{n}=P_{D}\left(P_{D}\left(L x_{n}\right)-\mu_{n} B\left(u_{n}\right)\right)$, and $z_{n}=P_{C}\left(y_{n}-\lambda_{n} A t_{n}\right)$. Then the extragradient Algorithm 3.1 reduces to the Algorithm 4.1 and we get the conclusion from and Theorem 3.2.

\section{Numerical experiments}

In this section, we give examples and numerical results to support Theorem 3.2. In addition, we compare the introduced algorithm with the parallel extragradient algorithm, which was presented in [27].

We consider the bifunctions $f$ and $g$ which are given in the form of Nash-Cournot oligopolistic equilibrium models of electricity markets [15, 34],

$$
f(x, y)=(P x+Q y)^{T}(y-x), \quad \forall x, y \in \mathbb{R}^{k},
$$




$$
g(u, v)=(U u+V v)^{T}(v-u), \quad \forall u, v \in \mathbb{R}^{m}
$$

where $P, Q \in \mathbb{R}^{k \times k}$ and $U, V \in \mathbb{R}^{m \times m}$ are symmetric positive semidefinite matrices such that $P-Q$ and $U-V$ are positive semidefinite matrices. The bifunctions $f$ and $g$ satisfy conditions (A1)-(A4) (see [37]). Indeed, $f$ and $g$ are Lipshitz-type continuous with constants $c_{1}=c_{2}=\frac{1}{2}\|P-Q\|$ and $d_{1}=d_{2}=\frac{1}{2}\|U-V\|$, respectively. Notice that, if $b_{1}=\max \left\{c_{1}, d_{1}\right\}$ and $b_{2}=\max \left\{c_{2}, d_{2}\right\}$, then both bifunctions $f$ and $g$ are Lipshitz-type continuous with constants $b_{1}$ and $b_{2}$.

The following numerical experiments are written in Matlab R2015b and performed on a Desktop with Intel(R) Core(TM) i3 CPU M $390 @ 2.67$ GHz 2.67 GHz and RAM 4.00 GB.

Example 5.1 Let the bifunctions $f$ and $g$ be given as (5.1) and (5.2), respectively. We will be concerned with the following boxes: $C=\prod_{i=1}^{k}[-5,5], D=\prod_{j=1}^{m}[-20,20], \bar{C}=\prod_{i=1}^{k}[-3,3]$ and $\bar{D}=\prod_{j=1}^{m}[-10,10]$. The nonexpansive mappings $T: C \rightarrow C$ and $S: D \rightarrow D$ are given by $T=P_{\bar{C}}$ and $S=P_{\bar{D}}$, respectively. The contraction mapping $h: C \rightarrow C$ is a $k \times k$ matrix such that $\|h\|<1$, while the linear operator $L: \mathbb{R}^{k} \rightarrow \mathbb{R}^{m}$ is a $m \times k$ matrix.

In this numerical experiment, the matrices $P, Q, U$, and $V$ are randomly generated in the interval $[-5,5]$ such that they satisfy above required properties. Besides, the matrices $h$ and $L$ are randomly generated in the interval $\left(0, \frac{1}{k}\right)$ and $[-2,2]$, respectively. We randomly generated starting point $x_{1} \in \mathbb{R}^{k}$ in the interval $[-20,20]$ with the following control parameters: $\delta_{n}=\frac{1}{2\|L\|^{2}}, \alpha_{n}=\frac{1}{n+2}$ and $\mu_{n}=\lambda_{n}=\frac{1}{4 \max \left\{b_{1}, b_{2}\right\}}$. The following three cases of the control parameter $\beta_{n}$ are considered:

Case 1. $\beta_{n}=10^{-10}+\frac{1}{n+1}$.

Case 2. $\beta_{n}=0.5$.

Case 3. $\beta_{n}=0.99-\frac{1}{n+1}$.

Note that to obtain the vector $u_{n}$, in the Algorithm 3.1, we need to solve the optimization problem

$$
\arg \min \left\{\mu_{n} g\left(P_{D}\left(L x_{n}\right), u\right)+\frac{1}{2}\left\|u-P_{D}\left(L x_{n}\right)\right\|^{2}: u \in D\right\}
$$

which is equivalent to the following convex quadratic problem:

$$
\arg \min \left\{\frac{1}{2} u^{T} J u+K^{T} u: u \in D\right\}
$$

where $J=2 \mu_{n} V+I_{m}$ and $K=\mu_{n} U P_{D}\left(L x_{n}\right)-\mu_{n} V P_{D}\left(L x_{n}\right)-P_{D}\left(L x_{n}\right)$ (see [27]).

On the other hand, in order to obtain the vector $v_{n}$, we need to solve the following convex quadratic problem:

$$
\arg \min \left\{\frac{1}{2} u^{T} \bar{J} u+\bar{K}^{T} u: u \in D\right\}
$$

where $\bar{J}=J$ and $\bar{K}=\mu_{n} U u_{n}-\mu_{n} V u_{n}-P_{D}\left(L x_{n}\right)$. Similarly, to obtain the vectors $t_{n}$ and $z_{n}$, we have to consider the convex quadratic problems in the same way as in (5.3) and (5.4), respectively. We use the Matlab Optimization Toolbox to solve vectors $u_{n}, v_{n}, t_{n}$ and $z_{n}$. The Algorithm 3.1 is tested by using the stopping criterion $\left\|x_{n+1}-x_{n}\right\|<10^{-3}$. In Table 1 , we randomly take 10 starting points and the presented results are in average. 
Table 1 The numerical results for different parameter $\beta_{n}$ of Example 5.1

\begin{tabular}{|c|c|c|c|c|c|c|c|}
\hline \multicolumn{2}{|c|}{ Size } & \multicolumn{3}{|c|}{ Average times (sec) } & \multicolumn{3}{|c|}{ Average iterations } \\
\hline k & $m$ & Case 1 & Case 2 & Case 3 & Case 1 & Case 2 & Case 3 \\
\hline 5 & 10 & 1.399695 & 1.957304 & 6.356185 & 37 & 54 & 171 \\
\hline 10 & 5 & 2.168317 & 2.916557 & 6.551182 & 56 & 75 & 179 \\
\hline 20 & 50 & 2.834138 & 3.785376 & 8.711813 & 58 & 80 & 186 \\
\hline 50 & 20 & 5.292192 & 6.570650 & 10.418191 & 111 & 138 & 220 \\
\hline
\end{tabular}

Table 2 The numerical results for the split equilibrium problem of Example 5.2

\begin{tabular}{|c|c|c|c|c|c|}
\hline \multicolumn{2}{|c|}{ Size } & \multicolumn{2}{|c|}{ Average times (sec) } & \multicolumn{2}{|c|}{ Average iterations } \\
\hline k & $m$ & Algorithm 3.1 & PEA & Algorithm 3.1 & PEA \\
\hline 5 & 10 & 0.862125 & 0.983111 & 31 & 44 \\
\hline 10 & 5 & 1.037650 & 1.991282 & 36 & 83 \\
\hline 20 & 50 & 1.607701 & 2.618173 & 44 & 85 \\
\hline 50 & 20 & 2.937581 & 7.926821 & 80 & 258 \\
\hline
\end{tabular}

From Table 1, we may suggest that a smallest size of parameter $\beta_{n}$, as $\beta_{n}=10^{-10}+\frac{1}{n+1}$, provides less computational times and iterations than other cases.

Example 5.2 We consider the problem (1.3) when $T=I_{\mathbb{R}^{k}}$ and $S=I_{\mathbb{R}^{m}}$ are identity mappings on $\mathbb{R}^{k}$ and $\mathbb{R}^{m}$, respectively. It follows that the problem (1.3) becomes the split equilibrium problem which was considered in [27]. In this case, we compare the Algorithm 3.1 with the parallel extragradient algorithm (PEA), which was in [27, Corollary 3.1]. For this numerical experiment, we consider the problem setting and the control parameters as in Example 5.1, but only for the case of parameter $\beta_{n}$ is $10^{-10}+\frac{1}{n+1}$. The starting point $x_{1} \in \mathbb{R}^{k}$ is randomly generated in the interval $[-5,5]$. We compare Algorithm 3.1 with PEA by using the stopping criterion $\left\|x_{n+1}-x_{n}\right\|<10^{-3}$. In Table 2, we randomly take 10 starting points and the presented results are in average.

From Table 2, we see that both computational times and iterations of Algorithm 3.1 are less than those of PEA.

\section{Conclusions}

We introduce a new extragradient algorithm and its convergence theorem for the split equilibrium problems and split fixed point problems. We also apply the main result to the problem of split variational inequality problems and split fixed point problems. Some numerical example and computational results are provided for discussing the possible usefulness of the results which are presented in this paper. We would like to note that this paper convinces us to consider the future research directions, for example, to consider the convergence analysis and the more general cases of the problem (like the non-convex case) directions; one may see [22, 29, 33] for more inspiration.

\section{Acknowledgements}

The authors are grateful to anonymous referees for their comments and remarks which helped to improve the paper. Vahid Dadashi is supported by Sari Branch, Islamic Azad University.

Funding

This work is partially supported by Naresuan University. 
Authors' contributions

All authors contributed equally to the writing of this paper. All authors read and approved the final manuscript.

\section{Author details}

${ }^{1}$ Department of Mathematics, Faculty of Science, Naresuan University, Phitsanulok, Thailand. ${ }^{2}$ Centre of Excellence in Nonlinear Analysis and Optimization, Faculty of Science, Naresuan University, Phitsanulok, Thailand. ${ }^{3}$ Department of Mathematics, Sari Branch, Islamic Azad University, Sari, Iran.

\section{Publisher's Note}

Springer Nature remains neutral with regard to jurisdictional claims in published maps and institutional affiliations.

\section{Received: 5 November 2018 Accepted: 30 April 2019 Published online: 14 May 2019}

\section{References}

1. Anh, P.N.: Strong convergence theorems for nonexpansive mappings and Ky Fan inequalities. J. Optim. Theory Appl. 154, 303-320 (2012)

2. Anh, P.N.: A hybrid extragradient method extended to fixed point problems and equilibrium problems. Optimization 62, 271-283 (2013)

3. Anh, P.N., An, L.T.H.: The subgradient extragradient method extended to equilibrium problems. Optimization 64, 225-248 (2015)

4. Anh, P.N., Le Thi, H.A.: An Armijo-type method for pseudomonotone equilibrium problems and its applications. J. Glob. Optim. 57, 803-820 (2013)

5. Bauschke, H.H., Borwein, J.M.: On projection algorithms for solving convex feasibility problems. SIAM Rev. 38, 367-426 (1996)

6. Blum, E., Oettli, W.: From optimization and variational inequalities to equilibrium problems. Math. Stud. 63, 123-145 (1994)

7. Byrne, C., Censor, Y., Gibali, A., Reich, S.: The split common null point problem. J. Nonlinear Convex Anal. 13, 759-775 (2012)

8. Censor, Y., Bortfeld, T., Martin, B., Trofimov, A.: A unified approach for inversion problems in intensitymodulated radiation therapy. Phys. Med. Biol. 51, 2353-2365 (2006)

9. Censor, Y., Elfving, T.: A multiprojection algorithm using Bregman projections in a product space. Numer. Algorithms 8, 221-239 (1994)

10. Censor, Y., Elfving, T., Kopf, N., Bortfeld, T.: The multiple-sets split feasibility problem and its applications for inverse problems. Inverse Probl. 21, 2071-2084 (2005)

11. Censor, Y., Gibali, A., Reich, S.: The subgradient extragradient method for solving variational inequalities in Hilbert space. J. Optim. Theory Appl. 148, 318-335 (2011)

12. Censor, Y., Gibali, A., Reich, S.: Algorithms for the split variational inequality problem. Numer. Algorithms 59(2), 301-323 (2012)

13. Censor, Y., Segal, A.: The split common fixed point problem for directed operators. J. Convex Anal. 16, 587-600 (2009)

14. Combettes, P.L.: The convex feasibility problem in image recovery. In: Hawkes, P. (ed.) Advances in Imaging and Electron Physics, pp. 155-270. Academic Press, New York (1996)

15. Contreras, J., Klusch, M., Krawczyk, J.B.: Numerical solution to Nash-Cournot equilibria in coupled constraint electricity markets. IEEE Trans. Power Syst. 19, 195-206 (2004)

16. Dadashi, V.: Shrinking projection algorithms for the split common null point problem. Bull. Aust. Math. Soc. 96(2), 299-306 (2017)

17. Dadashi, V., Khatibzadeh, $\mathrm{H} .:$ On the weak and strong convergence of the proximal point algorithm in reflexive Banach spaces. Optimization 66(9), 1487-1494 (2017)

18. Dadashi, V., Postolache, M.: Hybrid proximal point algorithm and applications to equilibrium problems and convex programming. J. Optim. Theory Appl. 174, 518-529 (2017)

19. Dadashi, V., Postolache, M.: Forward-backward splitting algorithm for fixed point problems and zeros of the sum of monotone operators. Arab. J. Math. (2019). https://doi.org/10.1007/s40065-018-0236-2

20. Daniele, P., Giannessi, F., Maugeri, A.: Equilibrium Problems and Variational Models. Kluwer Academic, Dordrecht (2003)

21. Dinh, B.V., Kim, D.S.: Projection algorithms for solving nonmonotone equilibrium problems in Hilbert space. J. Comput. Appl. Math. 302, 106-117 (2016)

22. Gibali, A., Küfer, K.-H., Süss, P.: Successive linear programming approach for solving the nonlinear split feasibility problem. J. Nonlinear Convex Anal. 15, 345-353 (2014)

23. Goebel, K., Kirk, W.A.: Topics in Metric Fixed Point Theory. Cambridge Studies in Advanced Mathematics, vol. 28. Cambridge University Press, Cambridge (1990)

24. Goebel, K., Reich, S.: Uniform Convexity, Hyperbolic Geometry, and Nonexpansive Mappings. Dekker, New York (1984)

25. He, Z:: The split equilibrium problem and its convergence algorithms. J. Inequal. Appl. (2012) https://doi.org/10.1186/1029-242X-2012-162

26. Hieu, D.V., Muu, L.D., Anh, P.K.: Parallel hybrid extragradient methods for pseudomotone equilibrium problems and nonexpansive mappings. Numer. Algorithms 73, 197-217 (2016)

27. Kim, D.S., Dinh, B.V.: Parallel extragradient algorithms for multiple set split equilibrium problems in Hilbert spaces Numer. Algorithms 77, 741-761 (2018)

28. Kraikaew, R., Saejung, S.: On split common fixed point problems. J. Math. Anal. Appl. 415, 513-524 (2014)

29. Li, Z., Han, D., Zhang, W.: A self-adaptive projection-type method for nonlinear multiple-sets split feasibility problem. Inverse Probl. Sci. Eng. 21, 155-170 (2012)

30. Mainge, P.E.: Strong convergence of projected subgradient methods for nonsmooth and nonstrictly convex minimization. Set-Valued Anal. 16, 899-912 (2008) 
31. Moudafi, A.: The split common fixed-point problem for demicontractive mappings. Inverse Probl. 26, 055007 (2010)

32. Moudafi, A.: Split monotone variational inclusions. J. Optim. Theory Appl. 150, 275-283 (2011)

33. Penfold, S., Zalas, R., Casiraghi, M., Brooke, M., Censor, Y., Schulte, R.: Sparsity constrained split feasibility for dose-volume constraints in inverse planning of intensity-modulated photon or proton therapy. Phys. Med. Biol. 62 3599-3618 (2017)

34. Quoc, T.D., Anh, P.N. Muu, L.D.: Dual extragradient algorithms extended to equilibrium problems. J. Glob. Optim. 52 139-159 (2012)

35. Reich, S., Sabach, S.: Three strong convergence theorems regarding iterative methods for solving equilibrium problems in reflexive Banach spaces. Contemp. Math. 568, 225-240 (2012)

36. Suwannaprapa, M., Petrot, N., Suantai, S.: Weak convergence theorems for split feasibility problems on zeros of the sum of monotone operators and fixed point sets in Hilbert spaces. Fixed Point Theory Appl. 2017, 6 (2017)

37. Tran, D.Q., Muu, L.D., Nguyen, V.H.: Extragradient algorithms extended to equilibrium problems. Optimization 57, 749-776 (2008)

38. Tuyen, T.M.: A strong convergence theorem for the split common null point problem in Banach spaces. Appl. Math. Optim. (2017). https://doi.org/10.1007/s00245-017-9427-z

39. Tuyen, T.M., Ha, N.S.: A strong convergence theorem for solving the split feasibility and fixed point problems in Banach spaces. J. Fixed Point Theory Appl. 20, 140 (2018)

40. Tuyen, T.M., Ha, N.S., Thuy, N.T.T.: A shrinking projection method for solving the split common null point problem in Banach spaces. Numer. Algorithms (2018). https://doi.org/10.1007/s11075-018-0572-5

41. Vuong, P.T., Strodiot, J.J., Nguyen, V.H.: Extragradient methods and linear algorithms for solving Ky Fan inequalities and fixed point problems. J. Optim. Theory Appl. 155, 605-627 (2012)

42. Xu, H.K.: Iterative algorithm for nonlinear operators. J. Lond. Math. Soc. 2, 1-17 (2002)

\section{Submit your manuscript to a SpringerOpen ${ }^{\circ}$ journal and benefit from:}

- Convenient online submission

- Rigorous peer review

- Open access: articles freely available online

- High visibility within the field

- Retaining the copyright to your article

Submit your next manuscript at $\gg$ springeropen.com 